\title{
Correlation functions of the one-dimensional Hubbard model in a magnetic field
}

\author{
Holger Frahm* \\ Department of Physics, University of Virginia, Charlottesville, Virginia 22901 \\ V. E. Korepin \\ Institute for Theoretical Physics, State University of New York at Stony Brook, \\ Stony Brook, New York 11794-3840
}

(Received 24 August 1990)

\begin{abstract}
We present a general method for the calculation of correlation functions in the repulsive one-dimensional Hubbard model at less than half-filling in a magnetic field $h$. We describe the dependence of the critical exponents that drive their long-distance asymptotics on the Coulomb coupling, the density, and $h$. This dependence can be described in terms of a set of coupled Bethe-Ansatz integral equations. It simplifies significantly in the strong-coupling limit, where we give explicit formulas for the dependence of the critical exponents on the magnetic field. In particular, we find that at small field the functional dependence of the critical exponents on $h$ can be algebraic or logarithmic-depending on the operators involved. In addition, we evaluate the singularities of the Fourier images of the correlation functions. It turns out that switching on a magnetic field gives rise to singularities in the dynamic field-field correlation functions that are absent at $h=0$.
\end{abstract}

\section{INTRODUCTION}

Recently, the relevance of correlation effects in interacting electron systems to high- $T_{c}$ superconductivity has led to growing interest in the computation of correlation functions for the one-dimensional Hubbard model ${ }^{1-7}$ since it combines the essentials of correlated electrons on one hand with the attractive feature of complete integrability on the other.

As a number of other systems in one spatial dimension, the Hubbard model has a critical point at zero temperature. For these models correlation functions decay as powers of the distance asymptotically. The calculation of these powers - the critical exponents - is of major interest. For some of these systems, for example, spinless fermions and the spin- $\frac{1}{2}$ Heisenberg chain, this can be done within Haldane's Luttinger liquid approach, ${ }^{8-11}$ based on the fact that these models belong to the same universality class as the Gaussian model. ${ }^{12}$ Due to conformal invariance, ${ }^{13}$ the possible universality classes of critical theories are related to a single dimensionless number: the central charge $c$ of the underlying Virasoro algebra ( $c=1$ for the Gaussian model). Furthermore, the finitesize corrections in the spectra of these models are closely related to the scaling dimensions of the fields present in the theory, ${ }^{14,15}$ which in turn determine the critical exponents. Common to all of these systems is the property that they have a single critical degree of freedom only. As is known from Lieb and Wu's ${ }^{16}$ exact solution the Hubbard model does not belong to this class: in general, both charge- and spin-density waves are critical. Only in a sufficiently strong magnetic field-where the spindensity waves have a gap-or at half-filling-where the Hubbard model is insulating and the charge excitations are massive- the remaining critical degree of freedom can be described within the scheme outlined above.

Based on the Bethe-Ansat $z$ solution the finite-size corrections for the half-filled Hubbard model at zero magnetic field have been calculated analytically and the critical theory has been found to be described by a single Virasoro algebra with central charge $c=1 .{ }^{17}$ The Hubbard model with attractive interaction at arbitrary filling also has central charge $c=1$; the critical exponents depend on the density of electrons. ${ }^{18}$ The magnetic field dependence of the critical behavior in a theory of this type has first been investigated in the Heisenberg spin chain. ${ }^{19}$ The critical theory still corresponds to $c=1$. The scaling dimensions, however, are found to depend on the magnetic field.

For systems with more than one critical degree of freedom-the situation is more complicated: the understanding of this class of systems is based on exact finitesize calculations ${ }^{20,21}$ in models that are soluble by a hierarchy ${ }^{22}$ of Bethe Ansätze (the Hubbard model belongs to this class). It has been found that they can be understood as a direct product of Virasoro algebras each having central charge $c=1$. As in the one-component case this continues to hold in a generic situation, i.e., with external fields coupled to the critical degrees of freedom. ${ }^{21}$

Woynarovich has calculated the finite-size corrections in the spectrum of the Hubbard model at less than halffilling analytically. ${ }^{23}$ His results can be interpreted in the 
framework of conformal quantum field theory following the lines mentioned above: the critical theory describing both charge- and spin-density waves is based on a product of two Virasoro algebras each having central charge $c_{i}=1$. In a previous paper we have used these results to calculate the critical exponents for the Hubbard model. ${ }^{5}$ There we have shown that while the central charges are universal the critical exponents depend on all the system parameters, i.e., density (or, alternatively, the chemical potential), coupling constant, and the magnetic field. In the present paper we concentrate on the magnetic field dependence. It is found to be logarithmic in the generic case-very similar to the situation in the spin- $\frac{1}{2}$ Heisenberg model. ${ }^{19}$ In fact, this similarity has been exploited before to show that the small field magnetic susceptibility $\chi$ exhibits logarithmic singularities as $h \rightarrow 0$ and as a further consequence the specific heat coefficient is singular. ${ }^{24}$ Since the critical exponents are closely related to thermodynamic quantities such as $\chi$ it is not surprising that they show the same singular field dependence. ${ }^{5}$ For the full Hubbard model (that is, including charge degrees of freedom) we find an additional linear field dependence in the corresponding contributions to the critical exponents. This is much weaker than the logarithmic one and shows up in certain correlators only.

At this point we would like to emphasize the difference between our approach ${ }^{5}$ and that of several other authors: $:^{3,6,7}$ the exact solution of the model and, in particular, the spectrum of low-lying excitations ${ }^{23}$ show that the contributions of charge- and spin-density waves cannot be described by two independent effective Hamiltonians-charge- and spin-density waves do interact. This means that the spectrum of conformal operator dimensions in this model is determined by a $2 \times 2$ matrix (the so-called dressed charge matrix, see below) rather than two scalar coupling constants. At zero magnetic field this fact is obscured to some extent since the dressed charge matrix is found to be triangular and the nonzero off-diagonal element is just one-half of one of the diagonal ones. ${ }^{23}$ Due to this fact, the critical exponents obtained when starting from two independent critical theories ${ }^{3,6,7}$ coincide with the ones found on the basis of the exact finite-size corrections. ${ }^{5}$

In the presence of a magnetic field, however, this situation changes: no simple relation between different elements of the dressed charge matrix holds and, as we show in this paper, the functional dependence of the various matrix elements on the field is significantly different. An important consequence of this is the appearance of an additional singularity of the electronic field correlation function in momentum space if the magnetic field is switched on. We do not think that this effect can be obtained within an approach that starts from separate effective theories for charge- and spin-density waves, respectively.

The Hubbard model describes spin- $\frac{1}{2}$ electrons on the lattice. The electrons are described by canonical Fermi fields $\psi_{\sigma}, \psi_{\sigma}^{\dagger}$ and the Hamiltonian is given by the follow- ing expression:

$$
\begin{aligned}
\mathcal{H}= & -\sum_{j=1}^{N} \sum_{\sigma}\left(\psi_{j+1, \sigma}^{\dagger} \psi_{j, \sigma}+\psi_{j, \sigma}^{\dagger} \psi_{j+1, \sigma}\right) \\
& +4 u \sum_{j=1}^{N} n_{j \uparrow} n_{j \downarrow}+\mu \sum_{j=1}^{N}\left(n_{j \uparrow}+n_{j \downarrow}\right) \\
& -\frac{h}{2} \sum_{j=1}^{N}\left(n_{j \uparrow}-n_{j \downarrow}\right) .
\end{aligned}
$$

Here $n_{j, \sigma}=\psi_{j, \sigma}^{\dagger} \psi_{j, \sigma}$ is the number of spin $\sigma$ electrons at site $j, 4 u>0$ is the on-site Coulomb repulsion, $\mu$ is the chemical potential, and $h$ is an external magnetic field.

Our paper is organized as follows: In the following section we shall review the Bethe-Ansatz solution of the model (1.1) in the aspects relevant to the present work. In Sec. III we consider the strong-coupling limit $u \rightarrow \infty$. In this limit the dependence of the critical exponents on the magnetic field $h$ simplifies essentially. In particular, we investigate this dependence for small fields and close to the critical field $h_{c}$ where all the spins are aligned and the model becomes ferromagnetic. In Sec. IV we apply these results to compute the critical exponents for a few interesting correlation functions. In the final section we calculate the singularities in the Fourier images of these correlation functions.

\section{THE BETHE-ANSATZ SOLUTION FOR THE HUBBARD MODEL}

Lieb and $\mathrm{Wu}^{16}$ have constructed a complete set of eigenfunctions of the Hubbard Hamiltonian (1.1). In the thermodynamic limit the zero-temperature ground state of the model consists of two Fermi seas, characterized by distribution functions $\rho_{c}(k)$ of charges with "holon" momentum $k$ and $\rho_{s}(\lambda)$ of down spins with spin-wave ("spinon") rapidity $\lambda$. Lieb and Wu have written down integral equations describing this configuration:

$$
\rho_{c}(k)=\frac{1}{2 \pi}+\frac{\cos k}{2 \pi} \int_{-\lambda_{0}}^{\lambda_{0}} d \lambda K(\sin k-\lambda ; u) \rho_{s}(\lambda),
$$

$$
\begin{aligned}
\rho_{s}(\lambda)= & \frac{1}{2 \pi} \int_{-k_{0}}^{k_{0}} d k K(\lambda-\sin k ; u) \rho_{c}(k) \\
& -\frac{1}{2 \pi} \int_{-\lambda_{0}}^{\lambda_{0}} d \mu K(\lambda-\mu ; 2 u) \rho_{s}(\mu) .
\end{aligned}
$$

The kernels of these equations are given by

$$
K(x ; \alpha)=\frac{2 \alpha}{\alpha^{2}+x^{2}} .
$$

The values of $\lambda_{0}$ and $k_{0}$ are related to the number of electrons per lattice site $n_{c}=N_{c} / N$ and the magnetization $\mathcal{M}=\left(N_{\uparrow}-N_{\downarrow}\right) / 2 N\left(N_{\sigma}\right.$ is the number of spin- $\sigma$ 
electrons) by the following equations:

$$
\begin{aligned}
& \int_{-k_{0}}^{k_{0}} d k \rho_{c}(k)=\frac{N_{c}}{N} \equiv n_{c}, \\
& \int_{-\lambda_{0}}^{\lambda_{0}} d \lambda \rho_{s}(\lambda)=\frac{N_{s}}{N} \equiv \frac{1}{2} n_{c}-\mathcal{M} .
\end{aligned}
$$

Another way to describe the system is in terms of integral equations for excitation energies:

$$
\begin{aligned}
\varepsilon_{c}(k)= & \varepsilon_{c}^{(0)}(k)+\frac{1}{2 \pi} \int_{-\lambda_{0}}^{\lambda_{0}} d \lambda K(\sin k-\lambda ; u) \varepsilon_{s}(\lambda) \\
\varepsilon_{s}(\lambda)= & \varepsilon_{s}^{(0)}(\lambda)+\frac{1}{2 \pi} \int_{-k_{0}}^{k_{0}} d k \cos k K(\lambda-\sin k ; u) \varepsilon_{c}(k) \\
& -\frac{1}{2 \pi} \int_{-\lambda_{0}}^{\lambda_{0}} d \mu K(\lambda-\mu ; 2 u) \varepsilon_{s}(\mu)
\end{aligned}
$$

Here $\varepsilon_{c}(k)$ is the energy of a charge-density excitation with momentum $k, \varepsilon_{s}(\lambda)$ that of a spin-density wave with rapidity $\lambda$. The bare energies $\varepsilon_{c, s}^{(0)}$ are

$$
\varepsilon_{c}^{(0)}(k)=\mu-\frac{h}{2}-2 \cos k, \quad \varepsilon_{s}^{(0)}(\lambda)=h .
$$

The solutions of Eqs. (2.4) define the energy bands. The ground-state configuration corresponds to the filling of all states with $\varepsilon_{c}(k)<0$ and $\varepsilon_{s}(\lambda)<0$. Consequently, the conditions

$$
\varepsilon_{c}\left(k_{0}\right)=0, \quad \varepsilon_{s}\left(\lambda_{0}\right)=0
$$

provide another way to define the values of $k_{0}$ and $\lambda_{0}$ for the ground state for given magnetic field $h$ and chemical potential $\mu$. At zero field $h=0$ one finds $\lambda_{0}=\infty$, the critical field $h=h_{c}$ where all the spins are pointing up, corresponds to $\lambda_{0}=0$. From (2.6) it is found to be

$$
h_{c}=\frac{2 u}{\pi} \int_{-\pi n_{c}}^{\pi n_{c}} d k \cos k \frac{\cos k-\cos \pi n_{c}}{u^{2}+\sin ^{2} k} .
$$

At large coupling $u$ the value of $h_{c}$ scales like $1 / u$ :

$$
h_{c} \sim \frac{2}{u}\left(n_{c}-\frac{1}{2 \pi} \sin 2 \pi n_{c}\right)+O\left(\frac{1}{u^{3}}\right), \quad \text { for } u \gg 1 .
$$

The quantity that determines the critical exponents is the dressed charge matrix: $:^{5}$

$$
Z \equiv\left(\begin{array}{cc}
Z_{c c} & Z_{c s} \\
Z_{s c} & Z_{s s}
\end{array}\right)=\left(\begin{array}{l}
\xi_{c c}\left(k_{0}\right) \xi_{c s}\left(\lambda_{0}\right) \\
\xi_{s r}\left(k_{0}\right) \xi_{s s}\left(\lambda_{0}\right)
\end{array}\right)
$$

Here the matrix $\xi_{i j}$ is defined as the solution of the following integral equations: ${ }^{21,23,25}$

$$
\begin{aligned}
\xi_{c c}(k)=1 & +\frac{1}{2 \pi} \int_{-\lambda_{0}}^{\lambda_{0}} d \lambda \xi_{c s}(\lambda) K(\lambda-\sin k ; u) \\
\xi_{c s}(\lambda)= & \frac{1}{2 \pi} \int_{-k_{0}}^{k_{0}} d k \cos k \xi_{c c}(k) K(\sin k-\lambda ; u) \\
& -\frac{1}{2 \pi} \int_{-\lambda_{0}}^{\lambda_{0}} d \mu \xi_{c s}(\mu) K(\mu-\lambda ; 2 u) \\
\xi_{s c}(k)= & \frac{1}{2 \pi} \int_{-\lambda_{0}}^{\lambda_{0}} d \lambda \xi_{s s}(\lambda) K(\lambda-\sin k ; u) \\
\xi_{s s}(\lambda)= & 1+\frac{1}{2 \pi} \int_{-k_{0}}^{k_{0}} d k \cos k \xi_{s c}(k) K(\sin k-\lambda ; u) \\
& -\frac{1}{2 \pi} \int_{-\lambda_{0}}^{\lambda_{0}} d \mu \xi_{s s}(\mu) K(\mu-\lambda ; 2 u)
\end{aligned}
$$

Other quantities of relevance are the Fermi velocities $v_{c}$ and $v_{s}$ of charge- and spin-density waves:

$$
\begin{aligned}
& v_{c}=\frac{1}{2 \pi \rho_{c}\left(k_{0}\right)} \varepsilon_{c}^{\prime}\left(k_{0}\right)>0, \\
& v_{s}=\frac{1}{2 \pi \rho_{s}\left(\lambda_{0}\right)} \varepsilon_{s}^{\prime}\left(\lambda_{0}\right)>0 .
\end{aligned}
$$

and the Fermi momenta $\mathcal{P}_{F, \uparrow}$ and $\mathcal{P}_{F, \downarrow}$ for electrons with spin up and down, respectively, i.e.,

$$
\mathcal{P}_{F, \uparrow(\downarrow)}=\frac{1}{2}\left(\pi n_{c} \pm 2 \pi \mathcal{M}\right)
$$

We already mentioned in the Introduction that the critical behavior of the repulsive Hubbard model at less than filling $\left(n_{c}<1\right)$ is described by the direct product of two Virasoro algebras, each having central charge $c=1$. The corresponding conformal dimensions of the primary fields are given in terms of the elements of (2.9) $\mathrm{as}^{5}$

$$
\begin{aligned}
2 \Delta_{c}^{ \pm}(\Delta \mathbf{N}, \mathbf{D})= & \left(Z_{c c} D_{c}+Z_{s c} D_{s}\right. \\
& \left. \pm \frac{Z_{s s} \Delta N_{c}-Z_{c s} \Delta N_{s}}{2 \operatorname{det} Z}\right)^{2} \\
2 \Delta_{s}^{ \pm}(\Delta \mathbf{N}, \mathbf{D})= & \left(\begin{array}{l}
Z_{c s} D_{c}+Z_{s s} D_{s} \\
\left. \pm \frac{Z_{c c} \Delta N_{s}-Z_{s c} \Delta N_{c}}{2 \operatorname{det} Z}\right)^{2}
\end{array}\right.
\end{aligned}
$$

Here $\Delta N_{c}$ and $\Delta N_{s}$ are integers which describe the quantum numbers of the operators involved in the correlation functions considered, i.e., the change in the number of charges and down spins with respect to the ground state. $D_{c}$ and $D_{s}$ are integer or half integer depending on the parities of $\Delta N_{c}$ and $\Delta N_{s}$ : 


$$
D_{c}=\frac{\Delta N_{c}+\Delta N_{s}}{2} \bmod 1, \quad D_{s}=\frac{\Delta N_{c}}{2} \bmod 1 .
$$

Knowing the conformal dimensions (2.13) the longdistance asymptotics of zero-temperature correlation functions is then given as a sum of terms

$$
\frac{\exp \left(-2 i D_{c} \mathcal{P}_{F, \uparrow} x\right) \exp \left[-2 i\left(D_{c}+D_{s}\right) \mathcal{P}_{F, \downarrow} x\right]}{\left(x-i v_{c} \tau\right)^{2 \Delta_{c}^{+}}\left(x+i v_{c} \tau\right)^{2 \Delta_{c}^{-}}\left(x-i v_{s} \tau\right)^{2 \Delta_{s}^{+}}\left(x+i v_{s} \tau\right)^{2 \Delta_{s}^{-}}}
$$

(we use Euclidean time $\tau$ ). As discussed above, $\Delta N_{c}$ and $\Delta N_{s}$ are fixed by the correlator one considers while summation has to be performed with respect to $D_{c}$ and $D_{s}$. The leading term in the asymptotics is given by the term in this sum which minimizes $\Delta^{ \pm}$.

Conformal quantum field theory also describes the correlation functions at small nonzero temperatures. Their exponential decay is given by the same dimensions (2.13) (see Ref. 5).

\section{THE STRONG-COUPLING LIMIT}

To illustrate the dependence of the critical exponents on the magnetic field let us consider now the limit $u \rightarrow$ $\infty$. This simplifies the integral equations (2.1), (2.4), and (2.10) significantly. After proper rescaling they can be rewritten in the following way:

$$
\begin{aligned}
\rho_{s}(\lambda)= & \frac{n_{c}}{\pi} \frac{1}{1+\lambda^{2}}-\frac{1}{2 \pi} \int_{-\lambda_{0}}^{\lambda_{0}} d \mu K(\lambda-\mu ; 2) \rho_{s}(\mu) \\
& +O\left(\frac{1}{u^{2}}\right), \\
\varepsilon_{s}(\lambda)= & h-\frac{h_{c}}{1+\lambda^{2}}-\frac{1}{2 \pi} \int_{-\lambda_{0}}^{\lambda_{0}} d \mu K(\lambda-\mu ; 2) \varepsilon_{s}(\mu) \\
& +O\left(\frac{1}{u^{2}}\right), \\
\xi_{s s}(\lambda)= & 1+\frac{\sin k_{0}}{\pi u} \frac{2 Z_{s c}}{1+\lambda^{2}} \\
& -\frac{1}{2 \pi} \int_{-\lambda_{0}}^{\lambda_{0}} d \mu K(\lambda-\mu ; 2) \xi_{s s}(\mu)+O\left(\frac{1}{u^{2}}\right) .
\end{aligned}
$$

( $\lambda_{0}$ differs from its value used in the previous section by a factor of $u$.) At $u=\infty$ the value of the critical field $h_{c}$ (2.8) vanishes - an infinitesimal magnetic field is sufficient to magnetize the system completely. From (3.1) we see, however, that at first order in $1 / u$ we already obtain nontrivial behavior.

The density and energy of the charge-density waves are given by the following expressions:

$$
\rho_{c}(k)=\frac{1}{2 \pi}+\frac{\cos k}{2 \pi u} \int_{-\lambda_{0}}^{\lambda_{0}} d \lambda \frac{2 \rho_{s}(\lambda)}{1+\lambda^{2}}+O\left(\frac{1}{u^{2}}\right)
$$

for $k \leq k_{0}$

$$
\varepsilon_{c}(k)=2\left(\cos k_{0}-\cos k\right)+O\left(\frac{1}{u^{2}}\right) .
$$

For the calculation of the elements of the dressed charge matrix (2.9) we shall neglect the corrections in $1 / u$. These contributions can, however, be obtained from (3.1) and similar expressions for the other elements. (For $h=0$ they have been written down in Ref. 5.) In this approximation they are given by

$$
\begin{aligned}
& Z_{c c}=1, \quad Z_{c s}=0, \\
& Z_{s c}=\frac{1}{2 \pi} \int_{-\lambda_{0}}^{\lambda_{0}} d \lambda \xi_{s s}(\lambda) K(\lambda ; 1) .
\end{aligned}
$$

For vanishing magnetic field $\lambda_{0}=\infty$ and Eqs. (3.1) can be solved by Fourier transformation. This case has been discussed in great detail in our previous paper. ${ }^{5}$ The dressed charge matrix has been found to be

$$
Z=\frac{1}{2}\left(\begin{array}{cc}
2 & 0 \\
1 & \sqrt{2}
\end{array}\right)
$$

For small magnetic field (and $\lambda_{0}$ large but finite) one can use the Wiener-Hopf (WH) method as outlined in the Appendix together with the condition

$$
\varepsilon_{s}\left(\lambda_{0}\right)=0
$$

to find the field dependence of $\lambda_{0}$ :

$$
\lambda_{0}=\frac{2}{\pi} \ln \left(\frac{h_{0}}{h}\right), \quad h_{0}=\sqrt{\frac{\pi^{3}}{2 e}} h_{c} .
$$

(A similar dependence has been found for the isotropic spin- $\frac{1}{2}$ Heisenberg magnetic chain. ${ }^{19}$ ) For the magnetization $\mathcal{M}$ we obtain in an analogous calculation

$$
\mathcal{M}=\frac{2 n_{c}}{\pi^{2} h_{c}} h
$$

and for the elements of the dressed charge matrix in a small magnetic field:

$$
Z_{s s}=\frac{1}{\sqrt{2}}\left(1+\frac{1}{4 \ln \left(h_{0} / h\right)}\right)+O\left(\frac{1}{\left[\ln \left(h_{0} / h\right)\right]^{2}}\right) \text {. }
$$

To calculate the leading correction to $Z_{s c}$ for finite $\lambda_{0}$ we make use of the fact that Eq. (3.3) can be written alternatively as

$$
Z_{s c}=\frac{1}{n_{c}} \int_{-\lambda_{0}}^{\lambda_{0}} d \lambda \rho_{s}(\lambda)=\frac{1}{2}-\frac{\mathcal{M}}{n_{c}}
$$


[this is possible, since the kernel of the integral equations (3.1) is symmetric]. Hence, we obtain with (3.7)

$$
Z_{s c}=\frac{1}{2}-\frac{2}{\pi^{2}} \frac{h}{h_{c}}+O\left(\frac{h}{h_{c} \ln \left(h_{0} / h\right)}\right) .
$$

Note the different functional dependence on $h$ of $Z_{s s}$ and $Z_{\text {sc }}$ near $h=0$. This has an interesting consequence on the conformal dimensions (2.13) entering the expressions for the correlation functions:

$$
\begin{aligned}
2 \Delta_{c}^{ \pm}(\Delta \mathbf{N}, \mathbf{D})= & \left(D_{c}+\frac{1}{2} D_{s} \pm \frac{1}{2} \Delta N_{c}\right)^{2} \\
& -\frac{4 h}{\pi^{2} h_{c}}\left(D_{c}+\frac{1}{2} D_{s} \pm \frac{1}{2} \Delta N_{c}\right) D_{s}
\end{aligned}
$$

$$
\begin{aligned}
2 \Delta_{s}^{ \pm}(\Delta \mathbf{N}, \mathbf{D})= & \frac{1}{2}\left[D_{s} \pm\left(\Delta N_{s}-\frac{1}{2} \Delta N_{c}\right)\right]^{2} \\
& +\frac{1}{4 \ln \left(h_{0} / h\right)}\left[D_{s}^{2}-\left(\Delta N_{s}-\frac{1}{2} \Delta N_{c}\right)^{2}\right] .
\end{aligned}
$$

The magnetic field dependence of the critical dimensions for the charge excitations is much weaker than that of the spin excitations. This is not surprising since the magnetic field couples directly to the spin degree of freedom. In general, the exponents of equal time correlators (where only the sum of $\Delta_{c}$ and $\Delta_{s}$ enters) will be dominated by the latter; in time-dependent quantities, however, this effect should become observable.

As $h$ approaches the critical field (2.8) from below, i.e., near the ferromagnetic state, $\lambda_{0}$ vanishes like

$$
\lambda_{0}=\left(\frac{h_{c}-h}{h_{c}}\right)^{1 / 2}
$$

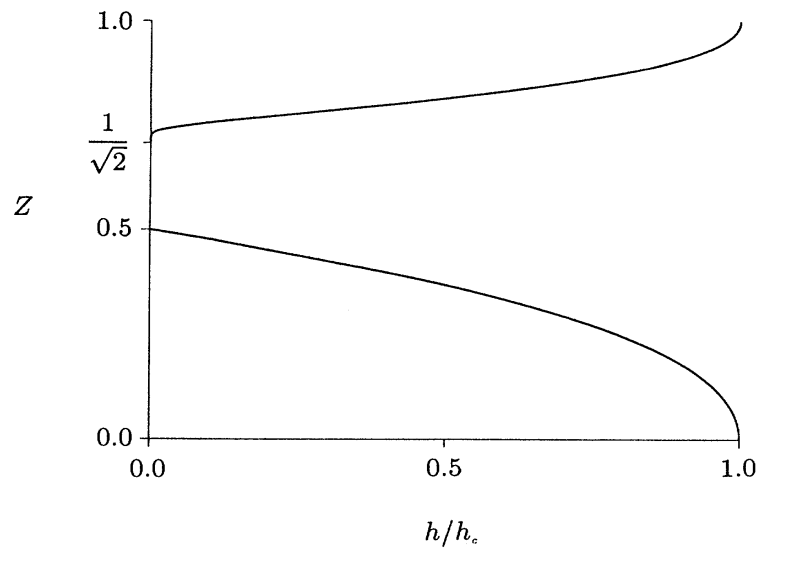

FIG. 1. Magnetic field dependence of the elements $Z_{s s}$ (upper curve) and $Z_{s c}$ (lower curve) of the dressed charge matrix in the strong-coupling limit. Note the drastically different functional behavior as $h \rightarrow 0$.

The dressed charge matrix in this regime is given by

$$
Z=\left(\begin{array}{ll}
1 & 0 \\
0 & 1
\end{array}\right)+\frac{1}{\pi}\left(\frac{h_{c}-h}{h_{c}}\right)^{1 / 2}\left(\begin{array}{cc}
0 & 0 \\
2 & -1
\end{array}\right)
$$

(this agrees with the limiting case $h=h_{c}$ considered in Ref. 5). The field dependence of the conformal dimensions to leading order is given by

$$
\begin{aligned}
& 2 \Delta_{c}^{ \pm}(\Delta \mathbf{N}, \mathbf{D})=\left(D_{c} \pm \frac{1}{2} \Delta N_{c}\right)^{2}+\frac{4}{\pi}\left(1-\frac{h}{h_{c}}\right)^{1 / 2}\left(D_{c} \pm \frac{1}{2} \Delta N_{c}\right) D_{s} \\
& 2 \Delta_{s}^{ \pm}(\Delta \mathbf{N}, \mathbf{D})=\left(D_{s} \pm \frac{1}{2} \Delta N_{s}\right)^{2}-\frac{2}{\pi}\left(1-\frac{h}{h_{c}}\right)^{1 / 2}\left(D_{s} \pm \frac{1}{2} \Delta N_{s}\right)\left[D_{s} \pm\left(\Delta N_{c}-\frac{1}{2} \Delta N_{s}\right)\right]
\end{aligned}
$$

At $h \geq h_{c}$ a phase transition similar to the one found at half-filling occurs: excitations with spin develop a gap and the corresponding contributions to the correlation functions decay like exponentials asymptotically.

In Fig. 1 we present numerical results based on Eqs. (3.1) and (3.3) for the magnetic field dependence of $Z_{s s}$ and $Z_{s c}$ for the entire region $0 \leq h \leq h_{c}$.

\section{CORRELATION FUNCTIONS IN THE STRONG-COUPLING LIMIT}

The results obtained in the last section can now be used to obtain the magnetic field dependence of the critical exponents of certain correlation functions. The basic procedure for this has been discussed in our earlier paper. ${ }^{5}$
First we consider the field-field correlation functions

$G_{\psi \psi}^{(\sigma)}(x, t)=\left\langle\psi_{\sigma}(x, t) \psi_{\sigma}^{\dagger}(0,0)\right\rangle, \quad \sigma=\uparrow, \downarrow$.

For $\sigma=\uparrow$ the quantum numbers of this operator are $\Delta N_{c}=1$ and $\Delta N_{s}=0$; from (2.14) we find that $D_{c}$ and $D_{s}$ both take half odd integer values. Hence the leading contribution with wave number $\mathcal{P}_{F, \uparrow}$ to $G_{\psi \psi}^{(\uparrow)}$ is

$$
\frac{\exp \left(i \mathcal{P}_{F, \uparrow} x\right)}{\left(x-i v_{c} \tau\right)^{2 \Delta_{c}^{+}}\left(x+i v_{c} \tau\right)^{2 \Delta_{c}^{-}}\left(x-i v_{s} \tau\right)^{2 \Delta_{s}^{+}}\left(x+i v_{s} \tau\right)^{2 \Delta_{s}^{-}}}
$$

where the expressions for the $\Delta_{c, s}^{ \pm}$are given by Eq. (2.13) with $\Delta N_{c}=1, \Delta N_{s}=0, D_{c}=-D_{s}=-\frac{1}{2}$. Near $h=0$ these expressions simplify considerably due to the simpler 
structure of the $Z$ matrix. We obtain

$$
\begin{aligned}
& 2 \Delta_{c}^{+}=\frac{1}{16}-\frac{h}{2 \pi^{2} h_{c}}, 2 \Delta_{c}^{-}=\frac{9}{16}+\frac{3 h}{2 \pi^{2} h_{c}}, \\
& 2 \Delta_{s}^{+}=\frac{1}{2}\left(\frac{1}{4 \ln \left(h_{0} / h\right)}\right)^{2}, \\
& 2 \Delta_{s}^{-}=\frac{1}{2}+\frac{1}{2}\left(\frac{1}{4 \ln \left(h_{0} / h\right)}\right)^{2} .
\end{aligned}
$$

The logarithmic field dependence of $\Delta_{s}^{ \pm}$cancels to first order. Fortunately, the next order is completely fixed by the leading correction to $Z_{s s}$ (3.8).

As $h$ approaches $h_{c}$ one obtains for this contribution

$$
\begin{aligned}
& 2 \Delta_{c}^{+}=0, \quad 2 \Delta_{c}^{-}=1-\frac{2}{\pi}\left(1-\frac{h}{h_{c}}\right)^{1 / 2}, \\
& 2 \Delta_{s}^{+}=\frac{1}{4}-\frac{3}{2 \pi}\left(1-\frac{h}{h_{c}}\right)^{1 / 2}, \\
& 2 \Delta_{s}^{-}=\frac{1}{4}+\frac{1}{2 \pi}\left(1-\frac{h}{h_{c}}\right)^{1 / 2} .
\end{aligned}
$$

[Here we neglect contributions of order $O\left(1-h / h_{c}\right)$.]

There is also a contribution with wave number $\mathcal{P}_{F, \uparrow}+$ $2 \mathcal{P}_{F, \downarrow}$ (corresponding to $D_{c}=D_{s}=-\frac{1}{2}$ ) with

$$
\begin{aligned}
& 2 \Delta_{c}^{+}=\frac{1}{16}-\frac{h}{2 \pi^{2} h_{c}}, 2 \Delta_{c}^{-}=\frac{25}{16}-\frac{5 h}{2 \pi^{2} h_{c}} \\
& 2 \Delta_{s}^{+}=\frac{1}{2}+\frac{1}{2}\left(\frac{1}{4 \ln \left(h_{0} / h\right)}\right)^{2} \\
& 2 \Delta_{s}^{-}=\frac{1}{2}\left(\frac{1}{4 \ln \left(h_{0} / h\right)}\right)^{2}
\end{aligned}
$$

for small magnetic field $h \ll h_{c}$ (again the corrections of order $\left[\ln \left(h_{0} / h\right)\right]^{-1}$ cancel). As $h$ approaches $h_{c}$ one obtains for this contribution

$$
\begin{aligned}
& 2 \Delta_{c}^{+}=0, \quad 2 \Delta_{c}^{-}=1+\frac{2}{\pi}\left(1-\frac{h}{h_{c}}\right)^{1 / 2}, \\
& 2 \Delta_{s}^{+}=\frac{1}{4}+\frac{1}{2 \pi}\left(1-\frac{h}{h_{c}}\right)^{1 / 2}, \\
& 2 \Delta_{s}^{-}=\frac{1}{4}-\frac{3}{2 \pi}\left(1-\frac{h}{h_{c}}\right)^{1 / 2} .
\end{aligned}
$$

For the spin-down field correlator we have $\Delta N_{c}=1$, $\Delta N_{s}=1$ and $D_{c}$ now runs through all integer, $D_{s}$ through all half odd integer numbers. The leading terms in the correlation functions are the ones given above for the spin-up correlators with $\mathcal{P}_{F, \uparrow}$ and $\mathcal{P}_{F, \downarrow}$ interchanged. For small magnetic field the dimensions are the ones given in Eqs. (4.3) and (4.5) with $h$ replaced by $-h$ in the expressions for $\Delta_{c}^{ \pm}$. For $h \rightarrow h_{c}$ the exponents for the contribution with wave number $\mathcal{P}_{F, \downarrow}\left(D_{c}=0, D_{s}=-\frac{1}{2}\right)$ are

$$
\begin{aligned}
& 2 \Delta_{c}^{ \pm}=\frac{1}{4} \mp \frac{1}{\pi}\left(1-\frac{h}{h_{c}}\right)^{1 / 2}, 2 \Delta_{s}^{+}=0, \\
& 2 \Delta_{s}^{-}=1-\frac{2}{\pi}\left(1-\frac{h}{h_{c}}\right)^{1 / 2},
\end{aligned}
$$

and for the component with wave number $2 \mathcal{P}_{F, \uparrow}+\mathcal{P}_{F, \downarrow}$ $\left(D_{c}=-1, D_{s}=\frac{1}{2}\right)$ we find

$$
\begin{aligned}
& 2 \Delta_{c}^{+}=\frac{1}{4}-\frac{1}{\pi}\left(1-\frac{h}{h_{c}}\right)^{1 / 2}, \\
& 2 \Delta_{c}^{-}=\frac{9}{4}-\frac{3}{\pi}\left(1-\frac{h}{h_{c}}\right)^{1 / 2}, \\
& 2 \Delta_{s}^{+}=1-\frac{2}{\pi}\left(1-\frac{h}{h_{c}}\right)^{1 / 2}, 2 \Delta_{s}^{-}=0 .
\end{aligned}
$$

Another correlation function of interest is the densitydensity correlation function

$$
\begin{aligned}
& G_{n n}(x, t)=\langle n(x, t) n(0,0)\rangle, \\
& n(x, t)=n_{\uparrow}(x, t)+n_{\downarrow}(x, t) .
\end{aligned}
$$

This operator has quantum numbers $\Delta N_{c}=\Delta N_{s}=0$ and $D_{c}, D_{s}$ take integer values. The leading contributions to the asymptotics apart from the constant are found to have wave number $2 \mathcal{P}_{F, \uparrow}$ (corresponding to $\left.D_{c}=-D_{s}=-1\right)$. For small magnetic field the corresponding critical dimensions are

$$
2 \Delta_{c}^{ \pm}=\frac{1}{4}+\frac{2 h}{\pi^{2} h_{c}}, \quad 2 \Delta_{s}^{ \pm}=\frac{1}{2}+\frac{1}{4 \ln \left(h_{0} / h\right)},
$$

The contribution with wave number $2 \mathcal{P}_{F, \downarrow}\left(D_{c}=0\right.$ and $D_{s}=-1$ ) has the same dimensions with $h$ in the expression for $\Delta_{c}^{ \pm}$replaced by $-h$. At $h=0$ the amplitudes of these contributions are known to be vanishing in the strong-coupling limit. ${ }^{1}$

As $h \rightarrow h_{c}$ the leading terms beyond the constant in the asymptotics of the density-density correlation function are found at zero wave number:

$$
\frac{1}{\left(x-i v_{c} \tau\right)^{2}}+\frac{1}{\left(x+i v_{c} \tau\right)^{2}}+\frac{1}{\left(x-i v_{s} \tau\right)^{2}}+\frac{1}{\left(x+i v_{s} \tau\right)^{2}}
$$

and at wave number $2\left(\mathcal{P}_{F, \uparrow}+\mathcal{P}_{F, \downarrow}\right)\left(D_{c}=-1, D_{s}=0\right)$ : the dimensions do not depend on the magnetic field to leading order. At infinite coupling $u$ they are known to be $2 \Delta_{c}^{ \pm}=1$ and $2 \Delta_{s}^{ \pm}=0 .^{5}$

The longitudinal spin-spin correlation function

$$
G_{\sigma \sigma}^{z}(x, t)=\left\langle S^{z}(x, t) S^{z}(0,0)\right\rangle
$$

(where $S^{z}(x, t)=\left[n_{\uparrow}(x, t)-n_{\downarrow}(x, t)\right] / 2$ ) has the same quantum numbers as the density-density correlator. Hence, the leading term beyond the constant is the one with wave number $2 \mathcal{P}_{F, \uparrow}$ and dimensions (4.10). The contribution of the spin-density waves $\Delta_{s}^{ \pm}$is the same as 
the one found in the isotropic Heisenberg chain. ${ }^{19}$ Finally we want to consider the transversal spin-spin correlation function

$$
G_{\sigma \sigma}^{\perp}(x, t)=\left\langle S^{-}(x, t) S^{+}(0,0)\right\rangle,
$$

with $S^{+}(x, t)=\psi_{\uparrow}^{\dagger}(x, t) \psi_{\downarrow}(x, t)$. The leading term in the asymptotics has wave number $\mathcal{P}_{F, \uparrow}+\mathcal{P}_{F, \downarrow}$ and with the corresponding quantum numbers $\Delta N_{c}=0, \Delta N_{s}=1$, $D_{c}=-\frac{1}{2}$ and $D_{s}=0$ we find for the dimensions in the strong-coupling limit

$$
\begin{aligned}
& 2 \Delta_{c}^{ \pm}=\frac{1}{4}, \quad 2 \Delta_{s}^{ \pm}=\frac{1}{2}-\frac{1}{4 \ln \left(h_{0} / h\right)} \quad \text { for } h \rightarrow 0 \\
& 2 \Delta_{c}^{ \pm}=\frac{1}{4}, \quad 2 \Delta_{s}^{ \pm}=\frac{1}{4}+\frac{1}{2 \pi}\left(1-\frac{h}{h_{c}}\right)^{1 / 2} \quad \text { for } h \rightarrow h_{c}
\end{aligned}
$$

$$
g(x, \tau)=\frac{\exp \left(i k_{0} x\right)}{\left(x-i v_{c} \tau\right)^{2 \Delta_{c}^{+}}\left(x+i v_{c} \tau\right)^{2 \Delta_{c}^{-}}\left(x-i v_{s} \tau\right)^{2 \Delta_{s}^{+}}\left(x+i v_{s} \tau\right)^{2 \Delta_{s}^{-}}} .
$$

To compute the Fourier transforms

$$
\tilde{g}(k, \omega)=\int d x \int d t e^{-i(k x-\omega t)} g(x, t),
$$

we first have to rotate the time coordinate from Euclidean time $\tau$ to real time $t$. Analyticity requires

$$
\tau=i t+\operatorname{sgn}(t) \text {. }
$$

Equivalently, the correct regularization of the cuts in the complex time coordinate can be obtained by replacing $\tau=i t$ in (5.1) and giving an infinitesimal imaginary part to the velocity:

$$
v_{i} \rightarrow v_{i} e^{-i 0} \text {. }
$$

All the following equations are to be understood that way. This gives

$$
g(x, t)=\frac{\exp \left(i k_{0} x\right)}{\left(x+v_{c} t\right)^{2 \Delta_{c}^{+}}\left(x-v_{c} t\right)^{2 \Delta_{c}^{-}}\left(x+v_{s} t\right)^{2 \Delta_{s}^{+}}\left(x-v_{s} t\right)^{2 \Delta_{s}^{-}}} .
$$

As mentioned above, this expression holds asymptotically only. Nevertheless, it allows us to calculate the behavior of the Fourier transforms $\tilde{g}(k, \omega)$ near the singularities

$$
\omega= \pm v_{c, s}\left(k-k_{0}\right) \text {. }
$$

Standard methods yield

$$
\tilde{g}(k, \omega) \sim \begin{cases}\operatorname{const}\left[\omega \mp v_{c}\left(k-k_{0}\right)\right]^{2\left(\Delta_{s}^{+}+\Delta_{s}^{-}+\Delta_{c}^{ \pm}\right)-1} & \text { for } \omega \approx \pm v_{c}\left(k-k_{0}\right) \\ \operatorname{const}\left[\omega \mp v_{s}\left(k-k_{0}\right)\right]^{2\left(\Delta_{c}^{+}+\Delta_{c}^{-}+\Delta_{s}^{ \pm}\right)-1} & \text { for } \omega \approx \pm v_{s}\left(k-k_{0}\right) .\end{cases}
$$

This formula is applicable only if all the dimensions $\Delta_{i}^{ \pm}$ are nonzero. If one of them vanishes (as is the case for the field-field correlator at zero magnetic field) the corresponding singularity disappears, i.e., const $=0$.

Note that the integral in (5.2) is not absolutely convergent in general. To prove (5.7) mathematically one should consider the case where $\sum \Delta_{a}^{ \pm}>1$ and the sum of three of the $\Delta$ 's less than $\frac{1}{2}$ and then continue analytically to obtain this expression.
For the Fourier transform of equal time correlators, i.e., terms like

$$
\begin{aligned}
\tilde{g}(k) & =\int d x e^{-i k x} g\left(x, t=0^{+}\right) \\
& =\int d x \frac{e^{-i\left(k-k_{0}\right) x}}{(x-i 0)^{2 \Delta^{+}}(x+i 0)^{2 \Delta^{-}}}
\end{aligned}
$$

(here $\Delta^{ \pm}=\Delta_{c}^{ \pm}+\Delta_{s}^{ \pm}$), one has to consider $k>k_{0}$ and 
$k<k_{0}$ separately. Contour integration yields

$$
\frac{\tilde{g}\left(k_{0}+p\right)}{\tilde{g}\left(k_{0}-p\right)}=\frac{\sin 2 \pi \Delta^{-}}{\sin 2 \pi \Delta^{+}}=(-1)^{2 s}, \quad p>0
$$

where $s=\Delta_{+}-\Delta_{-}$is the conformal spin of the operator under consideration. From (2.13) it is clear that $2 s$ is always an integer. Using this we find that

$$
\begin{aligned}
& \tilde{g}\left(k \approx k_{0}\right) \sim\left[\operatorname{sgn}\left(k-k_{0}\right)\right]^{2 s}\left|k-k_{0}\right|^{\nu}, \\
& \nu=2\left(\Delta_{c}^{+}+\Delta_{c}^{-}+\Delta_{s}^{+}+\Delta_{s}^{-}\right)-1 .
\end{aligned}
$$

The extra sign will appear in correlation functions of Fermi fields, e.g., the field-field correlator (4.1).

The results (5.10) and (5.7) are very general and can be applied to any correlation function. ${ }^{5}$ To illustrate this let us consider here a few examples in the strong-coupling limit. $\mathcal{P}_{F, \uparrow}$

The static field-field correlator has a singularity at

$$
\begin{aligned}
& \tilde{G}_{\psi \psi}^{(\uparrow)}\left(k \approx \mathcal{P}_{F, \uparrow}\right) \sim \operatorname{sgn}\left(k-\mathcal{P}_{F, \uparrow}\right)\left|k-\mathcal{P}_{F, \uparrow}\right|^{\nu}, \\
& \nu=\frac{1}{8}+\frac{1}{\left[4 \ln \left(h_{0} / h\right)\right]^{2}} \quad \text { for } h \rightarrow 0, \\
& \nu=\frac{1}{2}-\frac{3}{\pi}\left(1-\frac{h}{h_{c}}\right)^{1 / 2} \quad \text { for } h \rightarrow h_{c} .
\end{aligned}
$$

The $h=0$ exponent $\nu=\frac{1}{8}$ for the strong-coupling limit has been obtained before. ${ }^{3-7}$ At zero magnetic field $\nu$ is a monotonous function of the coupling constant $u$ and goes to 0 as $u \rightarrow 0$. For large but finite $u$ the leading correction is 5

$$
\nu(h=0)=\frac{1}{8}-\frac{3 \ln 2}{4 \pi u} \sin \pi n_{c} .
$$

Another singularity is at $\mathcal{P}_{F, \uparrow}+2 \mathcal{P}_{F, \downarrow}$ (b is a positive constant):

$$
\begin{aligned}
& \tilde{G}_{\psi \psi}^{(\uparrow)}\left(k \approx \mathcal{P}_{F, \uparrow}+2 \mathcal{P}_{F, \downarrow}\right) \sim \operatorname{sgn}\left(k-\mathcal{P}_{F, \uparrow}-2 \mathcal{P}_{F, \downarrow}\right) \\
& \times\left|k-\mathcal{P}_{F, \uparrow}-2 \mathcal{P}_{F, \downarrow}\right|^{\nu} \text {, } \\
& \nu= \begin{cases}\frac{9}{8}+\frac{1}{\left[4 \ln \left(h_{0} / h\right)\right]^{2}} & \text { for } h \rightarrow 0, \\
\frac{1}{2}+\frac{1}{\pi}\left(1-\frac{h}{h_{c}}\right)^{1 / 2} & \text { for } h \rightarrow h_{c} .\end{cases}
\end{aligned}
$$

As mentioned above, the corresponding singularities for the spin-down correlation functions at small magnetic fields are obtained by replacing $\mathcal{P}_{F, \uparrow}$ with $\mathcal{P}_{F, \downarrow}$ and vice versa. Near $h=h_{c}$ one obtains

$$
\begin{aligned}
& \tilde{G}_{\psi \psi}^{(\downarrow)}\left(k \approx \mathcal{P}_{F, \downarrow}\right) \sim \operatorname{sgn}\left(k-\mathcal{P}_{F, \downarrow}\right)\left|k-\mathcal{P}_{F, \downarrow}\right|^{\nu} \\
& \nu=\frac{1}{2}-\frac{2}{\pi}\left(1-\frac{h}{h_{c}}\right)^{1 / 2}
\end{aligned}
$$

$$
\begin{aligned}
\tilde{G}_{\psi \psi}^{(\downarrow)}\left(k \approx 2 \mathcal{P}_{F, \uparrow}+\mathcal{P}_{F, \downarrow}\right) \sim & \operatorname{sgn}\left(k-2 \mathcal{P}_{F, \uparrow}-\mathcal{P}_{F, \downarrow}\right) \\
& \times\left|k-2 \mathcal{P}_{F, \uparrow}-\mathcal{P}_{F, \downarrow}\right|^{\nu}, \\
\nu=\frac{5}{2}-\frac{6}{\pi}\left(1-\frac{h}{h_{c}}\right)^{1 / 2} . &
\end{aligned}
$$

The singularities of the correlation function $G_{\psi \psi}^{(\uparrow)}(\omega, k)$ can be obtained from (5.7). For the contributions with wave number $k \approx \mathcal{P}_{F, \uparrow}$ we find

$$
\tilde{G}_{\psi \psi}^{(\uparrow)}(\omega, k) \sim\left[\omega-v_{c}\left(k-\mathcal{P}_{F, \uparrow}\right)\right]^{\nu},
$$

$$
\text { for } \omega \approx v_{c}\left(k-\mathcal{P}_{F, \uparrow}\right)
$$

with

$$
\begin{aligned}
& \nu=\left\{\begin{array}{l}
-\frac{7}{16}+\frac{1}{\left[4 \ln \left(h_{0} / h\right)\right]^{2}} \quad \text { as } h \rightarrow 0 \\
-\frac{1}{2}-\frac{1}{\pi}\left(1-\frac{h}{h_{c}}\right)^{1 / 2} \quad \text { as } h \rightarrow h_{c}
\end{array}\right. \\
& \tilde{G}_{\psi \psi}^{(\uparrow)}(\omega, k) \sim\left[\omega+v_{c}\left(k-\mathcal{P}_{F, \uparrow}\right)\right]^{\nu}, \\
& \text { for } \omega \approx-v_{c}\left(k-\mathcal{P}_{F, \uparrow}\right),
\end{aligned}
$$

with

$$
\begin{aligned}
& \nu= \begin{cases}\frac{1}{16}+\frac{1}{\left[4 \ln \left(h_{0} / h\right)\right]^{2}} \quad \text { as } h \rightarrow 0 \\
\frac{1}{2}-\frac{3}{\pi}\left(1-\frac{h}{h_{c}}\right)^{1 / 2} \quad \text { as } h \rightarrow h_{c}\end{cases} \\
& \tilde{G}_{\psi \psi}^{(\uparrow)}(\omega, k) \sim\left[\omega-v_{s}\left(k-\mathcal{P}_{F, \uparrow}\right)\right]^{\nu}, \\
& \text { for } \omega \approx v_{s}\left(k-\mathcal{P}_{F, \uparrow}\right),
\end{aligned}
$$

with

$$
\nu= \begin{cases}-\frac{3}{8}+\frac{1}{2\left[4 \ln \left(h_{0} / h\right)\right]^{2}} \quad \text { as } h \rightarrow 0, \\ \frac{1}{4}-\frac{7}{2 \pi}\left(1-\frac{h}{h_{c}}\right)^{1 / 2} & \text { as } h \rightarrow h_{c} .\end{cases}
$$

For the Hubbard model without a magnetic field these singularities have also been found by Ren and Anderson. ${ }^{7}$ For finite $h$ there appears an additional singularity at $\omega+v_{s}\left(k-\mathcal{P}_{F, \uparrow}\right) \approx 0$ (this effect exists at any value of 
the coupling $u$ ):

$$
\tilde{G}_{\psi \psi}^{(\uparrow)}(\omega, k) \sim\left[\omega+v_{s}\left(k-\mathcal{P}_{F, \uparrow}\right)\right]^{\nu}
$$

$$
\text { for } \omega \approx-v_{s}\left(k-\mathcal{P}_{F, \uparrow}\right)
$$

with

$$
\nu=\left\{\begin{array}{l}
\frac{1}{8}+\frac{1}{2\left[4 \ln \left(h_{0} / h\right)\right]^{2}} \quad \text { as } h \rightarrow 0, \\
\frac{1}{4}-\frac{3}{2 \pi}\left(1-\frac{h}{h_{c}}\right)^{1 / 2} \quad \text { as } h \rightarrow h_{c} .
\end{array}\right.
$$

At $h=0$ the amplitude of this contribution to $\tilde{G}_{\psi \psi}^{(\uparrow)}(k, \omega)$ vanishes since $\Delta_{s}^{+} \rightarrow 0$ in this limit. Similarly, there will appear an additional singularity at $\omega-v_{s}\left(k-\mathcal{P}_{F, \uparrow}-\right.$ $\left.2 \mathcal{P}_{F, \downarrow}\right) \approx 0$ in the presence of a magnetic field.

For the static spin-spin correlator (4.13) we find for the singularity near wave number $\mathcal{P}_{F, \uparrow}+\mathcal{P}_{F, \downarrow}$

$$
\tilde{G}_{\sigma \sigma}^{\perp}\left(k \approx \mathcal{P}_{F, \uparrow}+\mathcal{P}_{F, \downarrow}\right) \sim\left|k-\mathcal{P}_{F, \uparrow}-\mathcal{P}_{F, \downarrow}\right|^{\nu}
$$

where

$$
\nu=\left\{\begin{array}{l}
\frac{1}{2}-\frac{1}{2 \ln \left(h_{0} / h\right)} \text { for } h \rightarrow 0 \\
\frac{1}{\pi}\left(1-\frac{h}{h_{c}}\right)^{1 / 2} \text { for } h \rightarrow h_{c} .
\end{array}\right.
$$

\section{CONCLUSION}

In Ref. 5 and the current paper we give a complete description of correlation functions in the one-dimensional Hubbard model. Based on exact finite size calculations and the principles of conformal quantum field theory we find expressions for the critical exponents that describe the long-distance asymptotics of the correlation functions in coordinate space or, equivalently, the singularities of their Fourier transforms in momentum space. The values of the critical exponents are given in terms of the elements of the dressed charge matrix, which in turn is defined as the solution of a set of Bethe-Ansatz integral equations (2.10). The critical behavior depends on all the system parameters, i.e., the density of electrons, the coupling constant, and the magnetic field. In Ref. 5 we have concentrated on the dependence on the density and the coupling constant at zero field and at the critical field where the ground state becomes ferromagnetic. In the present work we have computed the magnetic field dependence of the critical exponents in the limit of strong coupling.

To conclude, we would like to emphasize again that the integral equations (2.10) fix the critical behavior for arbitrary values of the system parameters. The reason for studying limiting cases is to simplify the expressions for the critical exponents. Furthermore, the analysis of the critical behavior is not restricted to the correlation functions considered here. The method presented can be applied to arbitrary operators.

\section{ACKNOWLEDGMENTS}

V.E.K. gratefully acknowledges the hospitality of M. Fowler and the Physics Department (University of Virginia), where much of this work was performed. This work has been supported by the National Science Foundation (NSF) under Grant Nos. DMR-8810541 and PHY89-08495.

\section{APPENDIX: WIENER-HOPF METHOD FOR EQS. (3.1)}

In this appendix we review the Wiener-Hopf method used above to solve integral equations of the type

$$
f(\lambda)=f^{(0)}(\lambda)-\mathcal{K}_{\lambda_{0}} * f(\lambda)
$$

where $\mathcal{K}$ is an integral operator whose action is defined by

$$
\mathcal{K}_{\lambda_{0}} * f(\lambda)=\frac{1}{2 \pi} \int_{-\lambda_{0}}^{\lambda_{0}} d \mu K(\lambda-\mu ; 2) f(\mu)
$$

for large but finite $\lambda_{0}$.

First we extend the definition of the bare function $f^{(0)}$ and of the kernel $K$ to the entire real axis. Following Yang and $\mathrm{Yang}^{26}$ we rewrite (A1) as

$$
\begin{aligned}
\left(1+\mathcal{K}_{\infty}\right) * f(\lambda)=f^{(0)}(\lambda)+ & \frac{1}{2 \pi}\left(\int_{-\infty}^{-\lambda_{0}}+\int_{\lambda_{0}}^{\infty}\right) \\
& \times d \mu K(\lambda-\mu ; 2) f(\mu)
\end{aligned}
$$

The operator $\left(1+\mathcal{K}_{\infty}\right)$ can be inverted by Fourier transform. Denoting

$$
\left(1+\mathcal{K}_{\infty}\right)^{-1} \equiv 1-\overline{\mathcal{K}}
$$

the integral equation (A3) can be written as

$$
f(\lambda)=f_{\infty}(\lambda)+\frac{1}{2 \pi}\left(\int_{-\infty}^{-\lambda_{0}}+\int_{\lambda_{0}}^{\infty}\right) d \mu \bar{K}(\lambda-\mu) f(\mu)
$$

where $f_{\infty}$ is the solution of (A1) for $\lambda_{0}=\infty$ and $\bar{K}$ is the kernel of the integral operator $\overline{\mathcal{K}}$ introduced in Eq. (A4). In the system considered here it is given in terms of its Fourier transform by

$$
\bar{K}(\omega)=\frac{1}{1+\exp (2|\omega|)} .
$$

Using that $f(\lambda)$ is an even function of $\lambda$ and shifting the variables in (A5) $\lambda=\lambda_{0}+x$ we obtain finally 


$$
\begin{aligned}
f(x)= & f_{\infty}\left(\lambda_{0}+x\right)+\int_{0}^{\infty} d y \bar{K}(x-y) f(y) \\
& +\int_{0}^{\infty} d y \bar{K}\left(2 \lambda_{0}+x+y\right) f(y) .
\end{aligned}
$$

Note that $\bar{K}\left(2 \lambda_{0}+x\right)$ is $O\left(\lambda_{0}^{-2}\right)$ for large $\lambda_{0}$ and positive $x$. This fact allows us to treat the last integral in Eq. (A7) as a perturbation. To every order the resulting equations are of Wiener-Hopf type:

$$
g(x)-\int_{0}^{\infty} d y \bar{K}(x-y) g(y)=g^{(0)}(x) .
$$

Fourier transformation yields

$$
[1-\bar{K}(\omega)] g^{+}(\omega)+g^{-}(\omega)=g^{(0)}(\omega),
$$

where $g^{ \pm}(\omega)=\int d x \Theta( \pm x) g(x) \exp (i \omega x)$ are the parts of $g(\omega)$ that are analytic for $\pm \operatorname{Im}(\omega)>0[\Theta(x)$ is the step function]. The key to the solution of this equation is to find a decomposition of the kernel into factors $G^{ \pm}$that are analytic in the upper and lower complex $\omega$ plane, respectively:

$$
1-\bar{K}(\omega)=\left[G^{+}(\omega) G^{-}(\omega)\right]^{-1}, \quad \lim _{\omega \rightarrow \infty} G^{ \pm}(\omega)=1 .
$$

For the present problem that is straightforward:

$$
\begin{aligned}
{\left[G^{-}(\pi x)\right]^{-1} } & =\left[G^{+}(-\pi x)\right]^{-1} \\
& =\frac{1}{\sqrt{2 \pi}} \Gamma\left(\frac{1}{2}+i x\right)(i x)^{-i x} e^{i x} .
\end{aligned}
$$

Using this factorization Eq. (A9) becomes

$$
\left[G^{+}(\omega)\right]^{-1} g^{+}(\omega)+G^{-}(\omega) g^{-}(\omega)=Q^{+}(\omega)+Q^{-}(\omega)
$$

where $Q^{ \pm}(\omega)$ are analytic for $\pm \operatorname{Im}(\omega)>0$,

$$
Q^{+}(\omega)+Q^{-}(\omega)=G^{-}(\omega) g^{(0)}(\omega)
$$

The analytic properties of the functions involved allow for the solution of Eq. (A12):

$$
g^{+}(\omega)=G^{+}(\omega) Q^{+}(\omega)
$$

In terms of this solution we have

$$
\int_{0}^{\infty} d x g(x)=g^{+}(\omega=0), \quad g(x=0)=-i \lim _{\omega \rightarrow \infty} \omega g^{+}(\omega) .
$$

To illustrate the scheme outlined above we apply it to the calculation of the magnetic field dependence of $\lambda_{0}$ (3.6): starting from Eq. (3.1) for $\varepsilon_{s}$ we find

$$
Q^{+}(\omega)+Q^{-}(\omega)=G^{-}(\omega)\left(\pi h \delta(\omega)-e^{-i \omega \lambda_{0}} \frac{\pi h_{c}}{2 \cosh \omega}\right)
$$

From this we obtain

$$
\begin{aligned}
Q^{+}(\omega)= & -\frac{h}{2 i} \frac{G^{-}(0)}{\omega+i 0}+e^{-\pi \lambda_{0} / 2} \frac{\pi h_{c} G^{-}(-i \pi / 2)}{2 i(\omega+i \pi / 2)} \\
& +O\left(e^{-3 \pi \lambda_{0} / 2}\right) .
\end{aligned}
$$

The second equation in (A15) gives

$$
\varepsilon_{s}\left(\lambda_{0}\right)=\frac{h G^{-}(0)}{2}-e^{-\pi \lambda_{0} / 2} \frac{\pi h_{c} G^{-}(-i \pi / 2)}{2}
$$

Finally using (A11) and (3.5) we have the result (3.6).
*Address after April 1, 1991: Institut für Theoretische Physik, Universität Hannover, D-3000 Hannover 1, Federal Republic of Germany.

${ }^{1}$ M. Ogata and H. Shiba, Phys. Rev. B 41, 2326 (1990).

${ }^{2}$ A. Parola and S. Sorella, Phys. Rev. Lett. 64, 1831 (1990).

${ }^{3}$ H. J. Schulz, Phys. Rev. Lett. 64, 2831 (1990).

${ }^{4}$ N. Kawakami and S.-K. Yang, Phys. Lett. A 148, 359 (1990).

${ }^{5}$ H. Frahm and V. E. Korepin, Phys. Rev. B 42, 10553 (1990).

${ }^{6}$ A. M. Tsvelick (unpublished).

${ }^{7}$ Y. Ren and P. W. Anderson (unpublished).

${ }^{8}$ F. D. M. Haldane, Phys. Rev. Lett. 45, 1358 (1980); J. Phys. C 14, 2589 (1981).

${ }^{9}$ F. D. M. Haldane, Phys. Lett. 81A, 153 (1981).

${ }^{10}$ F. D. M. Haldane, Phys. Rev. Lett. 47, 1840 (1981).

${ }^{11}$ F. D. M. Haldane, in Electron Correlation and Magnetism in Narrow-Band Systems, edited by T. Moriya (SpringerVerlag, Berlin, 1981), p. 150.

${ }^{12}$ L. Kadanoff and A. C. Brown, Ann. Phys. (N.Y.) 121, 318 (1979).

${ }^{13}$ A. A. Belavin, A. M. Polyakov, and A. B. Zamolodchikov, Nucl. Phys. B241, 333 (1984).
14 J. L. Cardy, Nucl. Phys. B270 [FS16], 186 (1986).

${ }^{15}$ H. W. J. Blöte, J. L. Cardy, and M. P. Nightingale, Phys. Rev. Lett. 56, 742 (1986); I. Affleck, ibid. 56, 746 (1986).

${ }^{16}$ E. H. Lieb and F. Y. Wu, Phys. Rev. Lett. 20, 1445 (1968).

${ }^{17}$ F. Woynarovich and H.-P. Eckle, J. Phys. A 20, L443 (1987).

${ }^{18}$ N. M. Bogoliubov and V. E. Korepin, Mod. Phys. Lett. B 1, 349 (1988); Int. J. Mod. Phys. B 3, 427 (1989).

${ }^{19} \mathrm{~A}$. G. Izergin and V. E. Korepin, Pis'ma Zh. Eksp. Teor. Fiz. 42, 414 (1985) [JETP Lett. 42, 512 (1985)]; N. M. Bogoliubov, A. G. Izergin, and V. E. Korepin, Nucl. Phys. B275 [FS17], 687 (1986).

${ }^{20}$ H. J. de Vega, J. Phys. A 21, L1089 (1988); J. Suzuki, ibid. 21, L1175 (1988).

${ }^{21}$ A. G. Izergin, V. E. Korepin, and N. Yu. Reshetikhin, J. Phys. A 22, 2615 (1989).

${ }^{22}$ C. N. Yang, Phys. Rev. Lett. 19, 1312 (1967).

${ }^{23}$ F. Woynarovich, J. Phys. A 22, 4243 (1989).

${ }^{24} \mathrm{~K}$. Lee and P. Schlottmann, Phys. Rev. Lett. 63, 2299 (1989).

${ }^{25}$ H. Frahm and N.-C. Yu, J. Phys. A 23, 2115 (1990).

${ }^{26}$ C. N. Yang and C. P. Yang, Phys. Rev. 150, 327 (1966). 